\title{
Multizentrische Retikulohistiozytose - eine histiozytäre Form der Arthritis multilans
}

\section{Multicentric Reticulohistiocytosis - A histiocytic form of Arthritis Mutilans}

\author{
Autoren \\ O. Nestler, M. Kayser, L. Unger \\ Institut \\ Städtisches Klinikum Dresden-Friedrichstadt, I. Medizinische Klinik, Dresden
}

Schlüsselwörter
Multizentrische
Retikulohistiozytose
Arthritis mutilans
Osteolysen
Key words
multicentric
reticulohistiocytosis
arthritis mutilans
osteolysis

Bibliografie

Dol http://dx.doi.org/ 10.1055/s-0035-1548863

Online-Publikation: 7.5.2015

Akt Rheumatol 2016; 41: 499-501 @ Georg Thieme Verlag KG Stuttgart · New York ISSN 0341-051X

Korrespondenzadresse

\section{Dr. Olaf Nestler}

Städtisches Klinikum

Dresden-Friedrichstadt

I. Medizinische Klinik

Dresden

01067

Tel.: + 49/351/4801260

Fax: +49/351/480 1119

nestler-ol@KHDF.de

\section{Zusammenfassung \\ $\nabla$}

Seronegative Arthritiden mit mutilierender Komponente erfordern immer besondere Aufmerksamkeit und ein differenziertes diagnostisches Vorgehen. Dabei müssen auch seltene Erkrankungen wie eine Multizentrische Retikulohistiozytose in Betracht gezogen werden. Nach dem Nachweis einer solchen Erkrankung konnten wir einem Patienten nach dem Versagen von cDMARDs mittels Etanercept helfen.

\section{Anamnese}

$\nabla$

In unserer Klinik stellte sich ein männlicher 58-jähriger Patient wegen anhaltend hoher Entzündungszeichen vor. Diese gingen einher mit Gelenkschwellungen und Schmerzen an Fingern, Ellenbogen, Hüfte und Füßen. Es bestand ein nicht ganz typisches Raynaud-Phänomen der Zehen und Hände. Weiterhin beschrieb er Parästhesien im Bereich der Finger und Myalgien in den Unterarmen und Oberschenkeln. Auch Effloreszenzen mit Arm- und Stammbetonung mit leicht schuppender Oberfläche erwähnte er (० Abb. 1-5).

Ungefähr 6 Monate vor Aufnahme erhielt der Patient eine Hüft-TEP rechts aufgrund einer Femurkopfresektion 3 Monate davor. Diese erfolgte aufgrund einer Femurkopfnekrose. Histologisch sei eine chronische Entzündung nachweisbar gewesen. Die Inflammation wurde mittels Antibiotika behandelt, welche jedoch keinen Einfluss auf die erhöhten Entzündungsparameter hatte und mit einer Clostridien-assoziierten Enterokolitis einherging. Er beschrieb weiterhin einen Alkoholabusus bis ein Jahr vor der Femurkopfnekrose und einen Bandscheibenvorfall im Bereich L2/3 mit folgender OP 2011. Er nahm Vorsorgeuntersuchungen regelmäßig wahr. Bereits ambulante Kollegen testeten Prednisolon bezüglich der Polyarthritis. Unter hohen Dosen waren diese teil-

\section{Abstract \\ $\nabla$}

Seronegative arthritis with mutilating component always requires special attention and a sophisticated diagnostic approach. Rare diseases like the multicentric reticulohistiocytosis must be considered. After the detection of such a disease, we could help a patient with etanercept after failure of cDMARDs.

weise regredient. An medikamentös eingestellten Erkrankungen bestand lediglich ein arterieller Hypertonus.

\section{Diagnostik $\nabla$}

Bei Aufnahme zeigte sich eine deutliche Bewegungseinschränkung im Bereich der Ellenbogen und Hüften. Die Ellenbogen waren rechtsseitig betont geschwollen. Auffällig waren randbetonte, leicht schuppende makulopapulöse flächige Effloreszenzen am Unterarm rechts sowie ein geschwollenes DIP-Gelenk III rechts. Der weitere internistische Status war unauffällig.

Laborchemisch konnten die deutlich erhöhten Entzündungszeichen (BSG $75 \mathrm{~mm} / \mathrm{h}, \mathrm{CRP} 102 \mathrm{mg} / \mathrm{l}$ $(<5)$ ), im Differenzialblutbild eine deutliche Monozytose $(11 \%)$ bei geringer Lymphopenie $(8 \%)$, ein erhöhtes Fibrinogen $(6,52 \mathrm{~g} / \mathrm{l})$ und Gesamtbilibrubin $(21,4 \mu \mathrm{mol} / \mathrm{l})$ gesehen werden. Rheumafaktoren, Citrullin-, Vimentin-Antikörper, ANA und ENA-Differenzierung waren negativ, ebenso wie die Infektionsserologie für Tuberkulose, Hepatitis B/C und HIV. Auffällig war eine gesteigerte Immunglobulin G- und Leichtkettenproduktion bei normalem Quotienten. Die Differenzierung des Immunglobulin G zeigte leicht erhöhte Werte für IgG 1-3 und ein normwertiges IgG4. 


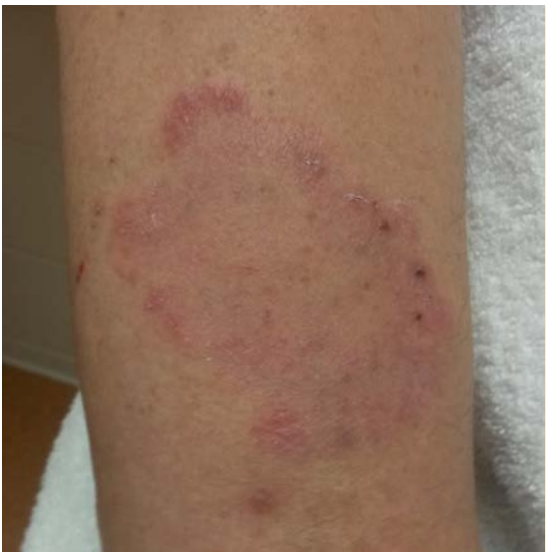

Abb. 1 Erythrosquamöse Effloreszenz rechter Unterarm.

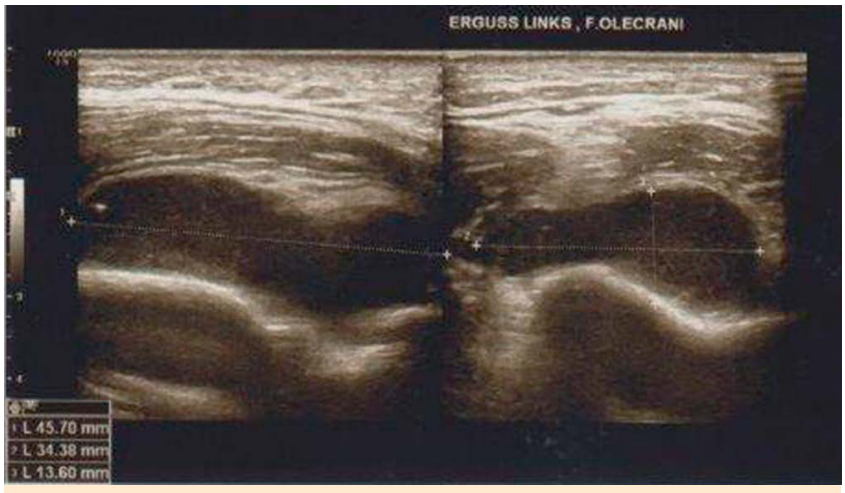

Abb. 2 Sonografie des rechten Ellenbogens mit ausgeprägtem Erguss.

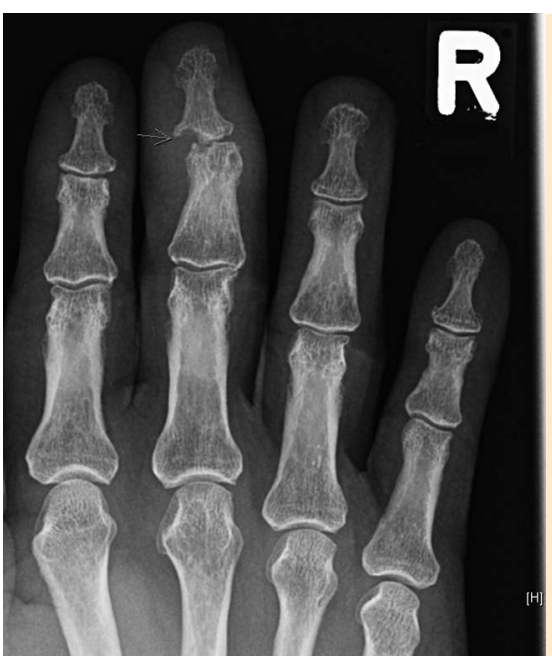

Abb. 3 Röntgenbild der rechten Hand, dorsovolar, mit Pfeil markierte Läsion am DIP 3.

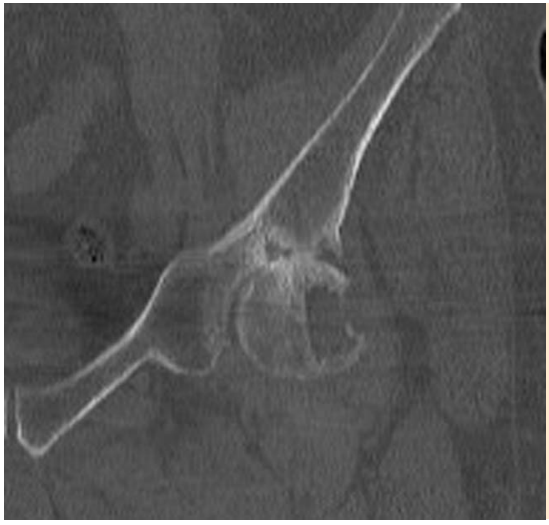

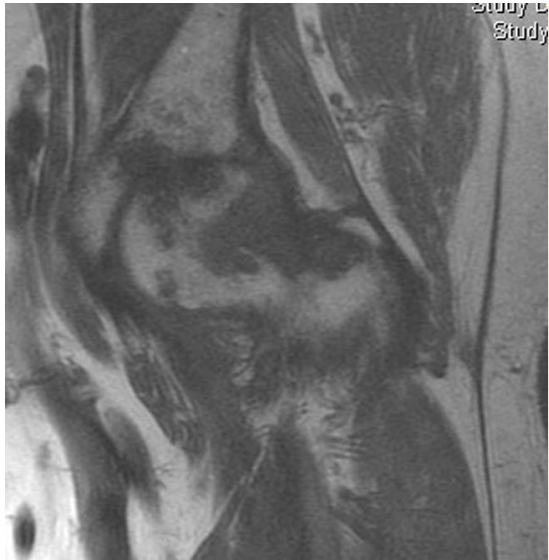

Abb. 5 MRT der linken Hüfte, COR FSE T1, ausgeprägte Knocheninfiltration im Bereich des Collum femoris

Es erfolgte ein umfangreiches Röntgen der schmerzenden Gelenke. Dabei zeigte sich ein ausgeprägter Erguss in beiden Ellenbogen und atypisch lokalisierte Usuren am distalen Interphalangealgelenk des Digitus III rechts, am Großzehengrundgelenk rechts als auch am Collum femoris sinister. Letztere erreichte einen Durchmesser von $16 \mathrm{~mm}$. Darüber hinaus gab es degenerative Veränderungen im Sinne einer Polyarthrose und lumbal betonte Spondylarthrosen. Ein Versuch den ausgeprägten Gelenkerguss $(46 \times 34 \times 14 \mathrm{~mm})$ im linken Ellenbogen zu punktieren scheiterte aufgrund der hohen Viskosität des Ergusses. Die veranlasste Knochenszintigrafie zur Detektion von weiteren Herden ergab einen erhöhten Knochenstoffwechsel mit Weichteilinfiltration im Bereich der 2013 implantierten Hüft-TEP. Eine durchgeführte MRT der großen Erosion im Femurhals links ergab den Verdacht auf eine frühe Hüftkopfnekrose bei Kapsulitis und Synovialitis.

Zur Beurteilung der weder sicher ekzematösen noch psoriatiformen Effloreszenzen führten wir eine Hautbiopsie durch. Dabei zeigte sich im Papillarkörper ein perivaskulär lokalisiertes lymphomonozytoides Infiltrat. Im Interstitium fanden sich schließlich mehrkernige Zellformen, die am ehesten histiozytären Ursprungs waren. Auch im Knochenmark konnten solche immunhistochemisch CD68 positiven, aber S100 negativen Zellen in erhöhter Zahl und Dichte nachgewiesen werden.

Die Kombination aus Infiltrateosinophilie, histiozytärer Anreicherung, atypisch lokalisierten ausgestanzten Knochenherden, Hautveränderungen und der chronische Verlauf wiesen den Weg zur Diagnose einer Multizentrischen Retikulohistiozytose. Die charakteristischen periungualen Papeln fehlten, jedoch bot das Exanthem eine ähnliche Struktur wie die typischen histologischen Beschreibungen.

Aufgrund der Prednislonsensibilität begannen wir eine cDMARD- Therapie mit MTX ohne nennenswerten Erfolg gefolgt von Leflunomid, das trotz deutlicher Besserung nicht ausreichte. Wegen der ausgeprägten entzündlichen und destruierenden Komponente entschlossen wir uns zur TNF-Inhibitor Gabe und begannen mit Etanercept. Es kam zu einem prompten Ansprechen. Nach mehr als einem halben Jahr ist der Patient weiterhin frei von Arthralgien, Hautveränderungen und Entzündungszeichen. Es bleibt der weitere Verlauf abzuwarten. Insbesondere war die große Osteolyse am linken Oberschenkelhals als kritisch zu betrachten. Im Rahmen der Implantation einer Hüft-TEP links im November 2014 wurde im Bereich der Osteolyse hier lediglich nekrotisches Material gefunden, eine histologische Sicherung der Diagnose gelang nicht. 


\section{Diskussion}

\section{(}

Die multizentrische Retikulohistiozytose wurde zuerst von Goltz und Laymon 1954 beschrieben [1]. Sie gehört zu den absoluten Raritäten. Genaue Zahlen finden sich in der Literatur nicht (jedoch Angaben von 1-2 Fällen auf eine Rheumatologenlaufbahn). Die Diagnose wird wie die Xanthomgruppe zum weiten Formenkreis der Non-Langerhans-Histiozytosen gezählt. Charakteristisch ist bei diesen Krankheiten, dass die Zellen weder S100 noch CD1a wie bei den zumeist letalen Formen der Langerhans-Histiozytosen exprimieren [2]. Es gelingt viel mehr der Nachweis von CD68-positiven Zellen, was die Erkrankung der histiozytären Zelllinie zuordnen lässt. Dies ist auch für die Diagnostik maßgebend. Es sollten Biopsien aus den betroffenen Hautarealen oder Synovialflüssigkeit entzündeter Gelenke gewonnen werden. In diesen lassen sich vermehrt mehrkernige Riesenzellen mit Lipideinlagerungen nachweisen, die ein eopsinophiles, mattes Zytoplasma enthalten. Diese sind TRAP- und PASpositiv und gehen typischerweise mit einem lymphozytären Infiltrat einher [3]. In Kombination mit den umliegenden Stromazellen, welche RANKL-postiv sind, werden die infiltrierenden Histiozyten zur osteoklastären Differenzierung stimuliert [2,3] und exprimieren Kathepsin K [4], das als Protease eine wesentliche Rolle im Knochenstoffwechsel spielt. Dies erklärt auch, warum es an den infiltrierten Stellen zu ausgestanzt anmutenden osteolytischen Befunden kommt, jedoch ohne generalisierte Osteopenie oder ausgeprägte Osteophytenbildung [4]. Jene Osteolysen sind typischerweise an den Gelenkrändern lokalisiert und der symmetrische Befall der DIP ist offenbar pathognomonisch [5]. Klinisch bildet sich das Bild einer Polyarthritis mit multiplem Gelenkbefall aus, das distale Gelenke präferiert. Darüber hinaus kommt es auch zu einer Hautbeteiligung. Immer wieder werden perlschnurartige Veränderungen-sogenannte „coral beads“ -im Bereich des Nagelbettes beschrieben [3]. Histologisch sind dies papulonoduläre indolente lymphomonozytoide Infiltrate, welche ebenso im Gesicht, periorbital oder an den Ellenbogen auftreten [6]. Daneben gibt es auch flächige Exantheme am Stamm und den Extremitäten [3]. Eine weitere Organbeteiligung wird in $20 \%$ der Fälle beschrieben und betrifft unter anderem die Lunge, den Gastrointestinaltrakt, die Nieren, die Speicheldrüsen, die Schilddrüse, das Herz oder die Leber. Das Krankheitsbild entwickeln in 75\% der Fälle Frauen mit einem Erkrankungsgipfel in der 4. Dekade. Dabei zeigen sich laborchemisch für gewöhnlich negative Rheumafaktoren, ANA und ein normaler Knochenstoffwechsel. Auffällig sind ausgeprägte Entzündungszeichen. Da die Erkrankung in bis zu 25\% der Fälle mit Malignomen assoziiert ist [6], sollte eine umfangreiche Diagnostik erfolgen. Bisher ist diese als Paraneoplasie im Rahmen von Melanomen, Sarkomen, Leukämien, Lymphomen und Karzinomen (u.a. Mamma, Kolon, Bronchus, Cervix, Magen, Ovarien) erwähnt. Weiterhin scheint es Overlap-Syndrome mit Autoimmunerkrankungen wie dem Systemischen Lupus erythematodes, dem Sjögren-Syndrom oder der Primär biliären Zirrhose [7] zu geben. Codriansky und Gorman $[4,6]$ beschreiben darüber hinaus auch einen Casus mit einer konkurrierenden Rheumatoiden Arthritis. Die Erkrankung wird mitunter als Psoriasisarthritis oder seronegative Rheumatoide Arthritis fehlgedeutet, aber auch die histizozytären Erkrankungen wie das Progressive noduläre Histiozytom (vornehmlicher Befall des Gesichts, keine Arthritis), das juvenile Xanthogranulom (Erkrankung des Jugendalters, Uveitis), und die nodulären Erkrankungen wie die lepromatöse Lepra, die tophöse Gicht, und die Sarkoidose müssen in Betracht gezogen werden. So schwierig sich die Diagnosefindung gestaltet, so heterogen sind auch die Behandlungsansätze. Es gibt weder einen Konsens noch Empfehlungen zur Therapie. Man weiß jedoch, dass die Erkrankung nach ca. 7 jahren selbstlimitiert, aber dies mit ausgeprägten Mutilationen einhergeht [8]. In der Literatur findet man Versuche mit NSAID und Kortikosteroiden. Es gibt auch Berichte mit alkylierenden Substanzen, Leflunomid und Methotrexat, jedoch ist das Outcome sehr unterschiedlich [6,9]. Aufgrund der osteoklastären Komponente nutzte man Bisphosphonate, welche in Kombination mit MTX zur Verbesserung in einzelnen Fällen führte [3,4]. Da in den ossären Läsionen bzw. in der Synovialflüssigkeit ein erhöhter Gehalt an TNF- $\alpha$ beschrieben ist, gab es auch erfolgreiche Versuche mit TNF-Inhibitoren [2,6,10]. Bei sehr eingeschränkter Literaturlage ist dennoch tendenziell erkennbar, dass bei hoher entzündlicher Aktivität eher die antiinflammatorische Behandlung günstiger ist, und bei geringen Entzündungszeichen die Gabe von Bisphosphonaten ausreichen kann. Kombinationstherapien aus TNF-Inhibitoren mit additiver anti-resorptiver Therapie sind aktuell nicht zu finden, bei entsprechender Indikation und Beschwerdepersistenz unter Monotherapie jedoch zu erwägen. Trotz der oben genannten Therapien sind die Verläufe vielfältig. Vereinzelt beschreiben Autoren ein Abheilen der Osteolysen, aber auch palliative handchirurgische Ansätze um ein Mindestmaß an Funktionsfähigkeit zu erhalten [10].

Ethik: Es liegt eine Zustimmung des Patienten zur Veröffentlichung vor.

Interessenkonflikt: Dr. Olaf Nestler: Teilnahmegebühren, Reisekosten, Vorträge - Abbvie, Pfizer, MSD, Roche, BMS, UCB

Dr. Marten Kayser: Teilnahmegebühren, Reisekosten, Vorträge Abbvie, Pfizer, MSD, Roche, BMS, UCB, Actelion

Dr. Leonore Unger: Referenten-Tätigkeit, Beratertätigkeit, Mitgliedschaft in advisory boards - Roche, Abbvie, Pfizer, MSD, UCB, GSK, Lilly, Amgen

\section{Literatur}

1 Goltz RW, Laymon CW. Multicentric reticulohistiocytosis of the skin and synovia: reticuolhistiocytoma or ganglioneuroma. AMA Archives of Dermatalogy and Syphilology 1954; 69: 717-731

2 Adamopoulos IE, Wordsworth PB, Edwards JR et al. Osteoclast differentiation and bon resoprtion in multicentric reticulohistiocytosis. Hum Pathol 2006; 37: 1176-1185

3 Goto $H$, Inaba M, Kobayashi $K$ et al. Successful treatment of multicentric reticulohistiocytosis with alendronate: evidence for a direct effect of bisühosphonate on histiocytosis. Arthritis Rheum 2003; 48: 3538-3541

4 Codriansky KA, Runger TM, Bhawan J et al. Multicentric reticulohistiocytosis: a systemic osteoclastic disease? Arthritis Rheum 2008; 59: 444-448

5 Santilli D, Lo Monaco A, Cavazzini PL et al. Multicentric reticulohistiocytosis: a rare cause of erosive arthropathy of the distal interphalangeal finger joint. Ann Rheum Dis 2002; 61: 485-487

6 Gorman JD, Danning C, Schumacher HR et al. Multicentric reticulhistiocytosis: a case report with immunohistochemical analysis and literature review. Arthritis Rheum 2000; 43: 930-938

7 Saito K, Fujii K, Awazu Y et al. A case of systemic lupus erythematosus complicated with multicentric reticulohistiocytosis (MRH): successful treatment of MRH and lupus with cyclosporin A. Lupus 2001; 10: $129-132$

8 Riaos Bianco JJ, Barbado Hernandez FJ, Gomez C et al. Multicentric reitculohistiocytosis. The long course of a rare disease. Scand J Rheumatol 2002; 31: 107-109

9 Gourmelen 0 , Le Loët X, Fortier-Beaulieu M et al. Methotrexate treatment of multicentirc reticulohistiocytosis. J Rheumatol 1991; 18: 627-628

10 Kovach BT, Calamia KT, WalshJS et al. Treatment of multicentric reticulohistiocytosis with etanercept. Arch Dermatol 2004; 140919-140921 